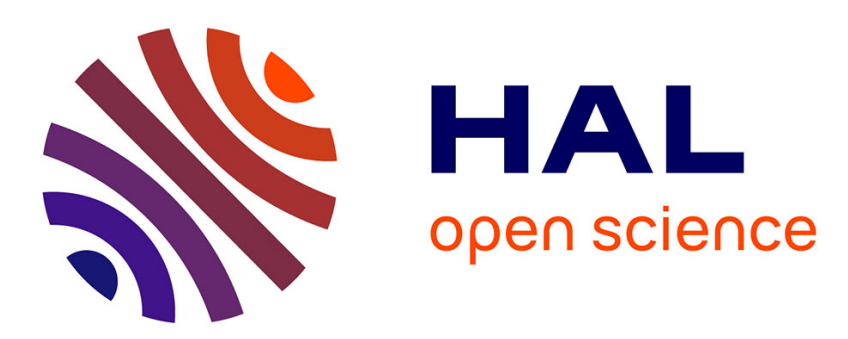

\title{
A numerical framework for the stability and cardinality analysis of concentric tube robots: Introduction and application to the follow-the-leader deployment
}

\author{
Quentin Peyron, Kanty Rabenorosoa, Nicolas Andreff, Pierre Renaud
}

\section{- To cite this version:}

Quentin Peyron, Kanty Rabenorosoa, Nicolas Andreff, Pierre Renaud. A numerical framework for the stability and cardinality analysis of concentric tube robots: Introduction and application to the follow-the-leader deployment. Mechanism and Machine Theory, 2018, 132, pp.176-192. 10.1016/j.mechmachtheory.2018.10.020 . hal-02126175

\section{HAL Id: hal-02126175 \\ https://hal.science/hal-02126175}

Submitted on 10 May 2019

HAL is a multi-disciplinary open access archive for the deposit and dissemination of scientific research documents, whether they are published or not. The documents may come from teaching and research institutions in France or abroad, or from public or private research centers.
L'archive ouverte pluridisciplinaire HAL, est destinée au dépôt et à la diffusion de documents scientifiques de niveau recherche, publiés ou non, émanant des établissements d'enseignement et de recherche français ou étrangers, des laboratoires publics ou privés. 


\title{
A numerical framework for the stability and cardinality analysis of concentric tube robots: Introduction and application to the follow-the-leader deployment
}

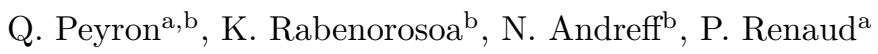 \\ ${ }^{a} I C U B E-A V R, U D S-C N R S-I N S A, 1$ Place de l'Hôpital, 67091 Strasbourg, France \\ ${ }^{b}$ FEMTO-ST Institute, Univ. Bourgogne Franche-Comté/CNRS, 25000 Besancon, France
}

\begin{abstract}
Concentric tube robot (CTR) is a promising class of continuum robots for medical interventions given their compactness and dexterity. Their dexterity is in particular being used to achieve so called Follow-the-Leader (FTL) deployments, where the tip path draws the shape of the robot. During this kind of deployment they can however be subject to elastic instabilities, and the number of reachable configurations may vary for a given state of actuators. These cardinality and stability changes need therefore to be predicted during CTR design. Available methods and results are limited, with restrictive assumptions on number and properties of tubes. We therefore propose in this paper a numerical framework for the cardinality and stability assessment of CTR. It is based on the association of dynamic relaxation, continuation method and bifurcation analysis. The numerical framework is validated by reproducing reference results on the stability and cardinality of two-tube robots. Then, new results on three-tube CTR deploying in a FTL manner are presented. The framework genericity allows in particular to provide new insights on the behaviour of CTR with helical-shaped tubes.
\end{abstract}

Keywords: Concentric tube robot, Continuation method, Stability evaluation, Follow-the-leader deployment, Bifurcation analysis

\section{Introduction}

Concentric tube robots (CTR) are telescopic assemblies of pre-curved tubes which are rotated and translated at their base. The superposition of their curvatures creates internal forces which change the equilibrium state of the robot. It is thus possible to control the shape and the tip position of this cannula robot. In addition, CTR can achieve, under certain conditions, deployments in a Follow-The-Leader (FTL) manner, i.e. with the tip path drawing the shape of the robot $[3,8]$. These features make this kind of manipulator of particular interest for navigation in narrow spaces. Therefore, they have been investigated extensively for minimally invasive interventions such as beating-heart surgery 
[11], vitro-retinal surgery [23] and nasal cavity exploration [9]. The reader may refer to the review paper [1] for more examples of CTR in medical applications.

Their use is however hampered by stability issues, due to the tube elastic interactions, which result in unsafe behavior of the robot. During a deployment, and particularly during FTL deployment where tubes can be placed in opposition, the tubes endure torsional solicitations and store elastic potential energy as described in [16, 6]. Webster et al. [16] and Dupont et al. [6] then showed that multiple equilibrium configurations with different energy levels can as a consequence exist for the same actuation inputs, which means the cardinality of the CTR kinematic model is greater than one. In the remainder of the paper, the number of equilibrium configurations existing for the same actuation inputs will be thus referred as the CTR cardinality. This property impacts the robot workspace and has therefore to be evaluated.

In addition, the energy stored in the CTR can be suddenly released, leading the robot to be unstable and to snap away from an equilibrium configuration with hazardous dynamics. Such snapping is unacceptable, especially in medical context. Works have therefore been devoted to the stability improvement of CTR through tube design [13,21] and path planning [22]. Conditions are then derived on design parameters like the number of tubes, tube geometry or deployment sequence. It is then mandatory to assess stability taking into account all these variables.

Assessment of stability and cardinality are complex and cumbersome tasks. The existing approaches $[6,15,14]$ use analytical manipulations of the kinematic model which are possible after several assumptions on tube number and properties. Results have been produced for two-tube CTR in free space with constant and planar pre-curvature of tubes. Dupont et al. [6] obtained the equations describing the static behavior of the robot according to its base actuation angles. This allowed them to establish a global stability criterion, ensuring that the robot is stable for any rotation of the tubes. This study was completed by Hendrick et al. [15] who obtained the kinematic model cardinality when two tubes are deployed and a local expression of the two possible equilibria using perturbation methods. Global [15] and local [14] stability criteria have been also established for robots with more than two tubes. The local approach in [14] is interesting for its genericity as it handles CTRs with arbitrary geometry and mechanical properties of the tubes. It however provides local stability evaluation only, and to our knowledge no other work provides cardinality evaluation for arbitrary number and properties of tubes.

As a summary, there are few results about the cardinality of CTR during their deployment, especially for the FTL case. Moreover there is no tool able to evaluate the robot cardinality and the equilibrium stability that remains applicable without limiting assumptions on the tube number and properties. We propose in this paper such a tool in the form of a numerical framework that can be applied to a generic kinematic model of CTR. It is based on the simulation of CTR deployment along paths, which consists in our approach to compute connected sets of equilibria, using dynamic relaxation and continuation methods [18]. Bifurcation analysis [19] is then used to evaluate robot cardinality 
without being dependent on tube number and properties. The kinematic model we use is based on the expression of the CTR potential energy. This enables one to assess numerically the local stability. The proposed framework is particularly adapted to this context, considering the successful previous use of the numerical tools in the close context of buckled beam stability analysis [20]. Use of continuation in robotics was indeed also advantageously considered for robot workspace analysis in [17] or [26].

This paper is then organized as followed. A generic kinematic model describing the behavior of a CTR is derived from its potential energy in Section 2. Transformation of this model through the different steps of the proposed numerical framework is detailed in Section 3. Reference results on two-tube CTR are generated in Section 4 to validate the proposed approach and to evaluate its performances. New results are finally produced in Section 5 for three-tube CTR, with constant and helical pre-curvature, deploying in a FTL manner.

\section{Background of CTR kinematics}

Our goal is to analyze the stability and the cardinality of any given CTR in terms of number of tubes and tube properties. This supposes to derive a kinematic model with adequate genericity. We present in this section such a model, which is based on the energy derivation presented in [29]. In this work, CTR are composed of arbitrary number of tubes, with arbitrary mechanical properties and planar pre-curvature. We add to this model the boundary conditions given in $[7,8]$ to take into account tubes with transmission lengths and helical initial shape. We use then the virtual tube method developed in [14] to facilitate the implementation of the model. Model parameters and equations used throughout this work are now introduced and developed in order to ease the understanding of the results.

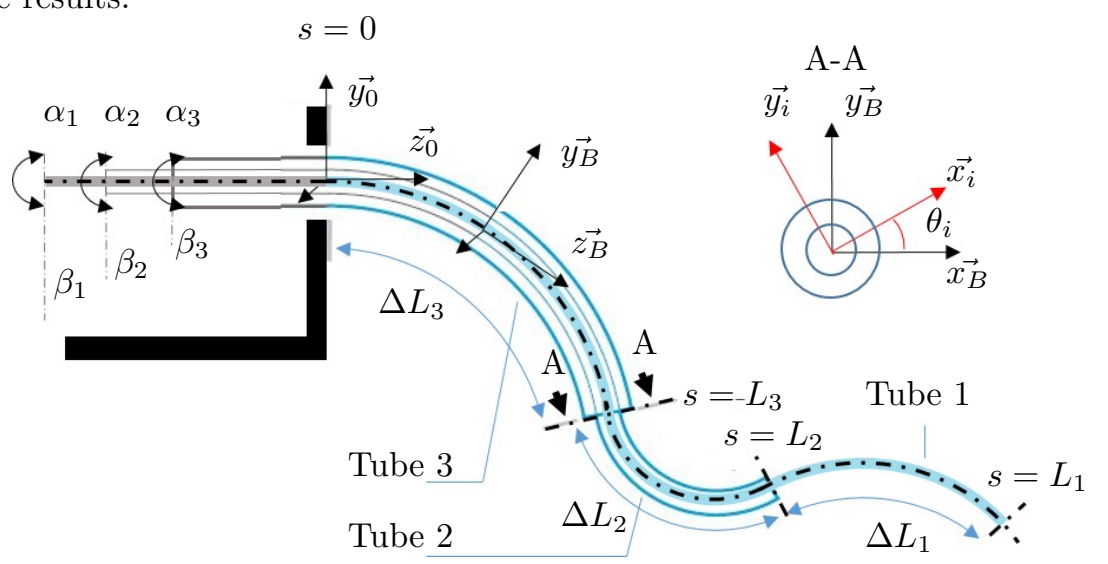

Figure 1: CTR schematics representation with parametrization. The figure corresponds to a 3 -tube CTR with 3 sections. The robot backbone is represented with a dash-dotted curve.

As illustrated in Figure 1, the CTR is composed of $n$ tubes. These tubes are 
numbered from the innermost to the outermost. The deployed length of tube $i$, which is its translatory degree of freedom, is denoted $L_{i}$. It is measured with respect to the initial configuration where all tube extremities are located at arc length $s=0$. The rotational degree of freedom of tube $i$ is denoted by the angle $\alpha_{i}$. The tubes are actuated in rotation and translation at the arc-length $s=-\beta_{i}$, where $\beta_{i}$ is the transmission length of tube $i$. The robot is considered as composed of $n$ sections, the number of tubes being constant along each one. The sections are indexed from the distal end to the proximal one, and the length of the $i$-th section is denoted $\Delta L_{i}$. The section lengths depend on the value of the deployed length of each tube. For example, in the configuration depicted on Figure 1, the section lengths are expressed by $\Delta L_{i}=L_{i}-L_{i+1}, i \in[1,3]$.

A base frame $\mathcal{R}_{0}=\left(O, \overrightarrow{x_{0}}, \overrightarrow{y_{0}}, \overrightarrow{z_{0}}\right)$ is attached to the base of the robot. The robot backbone is described by a curve composed by points of coordinate $\boldsymbol{p}(s)$. The local configuration of the robot can then be defined using a Bishop frame $\mathcal{R}_{B}=\left(\boldsymbol{p}(s), \overrightarrow{x_{B}}, \overrightarrow{y_{B}}, \overrightarrow{z_{B}}\right)$, obtained by sliding $\mathcal{R}_{0}$ along the backbone without any rotation about its tangent. A frame $\mathcal{R}_{i}=\left(\boldsymbol{p}(s), \overrightarrow{x_{i}}, \overrightarrow{y_{i}}, \overrightarrow{z_{i}}\right)$ is then attached to each tube $i$, that is obtained by applying a rotation $\left(\theta_{i}(s), \overrightarrow{z_{B}}\right)$ to $\mathcal{R}_{B}$, with $\theta_{i}(s)$ the twist angle of tube $i$ at arc length $s$. In the remainder of this paper, we will use when needed subscripts $(0, B$ and $i)$ to indicate the frame in which vectors are projected.

The curvature of a CTR is represented by the Darboux vector $\boldsymbol{u}(s)$ of the curve drawn by its backbone [6]. This vector gathers the angular rate of the frame $\mathcal{R}_{B}$ according to $s$ and projected in one of the previously defined frames. The pre-curvature of tube $i$ is denoted $\hat{\boldsymbol{u}}_{\boldsymbol{i}}(s)$ and is expressed in $\mathcal{R}_{i}$. The mechanical properties of tube $i$ are described by bending and torsional stiffnesses $k_{i b}(s)$ and $k_{i t}(s)$. They are gathered in a stiffness matrix $\boldsymbol{K}_{\boldsymbol{i}}(s)$ expressed in $\mathcal{R}_{i}$ such that:

$$
\boldsymbol{K}_{\boldsymbol{i}}(s)=\left[\begin{array}{ccc}
k_{i b}(s) & 0 & 0 \\
0 & k_{i b}(s) & 0 \\
0 & 0 & k_{i t}(s)
\end{array}\right]
$$

The dependences in $s$ are not mentioned in the following of the paper for the sake of compactness. Let us consider the $j$-th section of the CTR where $m$ tubes interact with each other. The potential energy of this section of the CTR is written [29]:

$$
E=\frac{1}{2} \int_{s=L_{j}}^{L_{j+1}} \sum_{i=1}^{m}\left(\boldsymbol{u}_{\boldsymbol{i}}-\hat{\boldsymbol{u}_{\boldsymbol{i}}}\right)^{T} \boldsymbol{K}_{\boldsymbol{i}}\left(\boldsymbol{u}_{\boldsymbol{i}}-\hat{\boldsymbol{u}_{\boldsymbol{i}}}\right) d s
$$

The telescopic assembly of the tubes imposes that the tubes share the same bending curvature. The Darboux vectors of each tube, projected in the $\mathcal{R}_{B}$ frame, are thus equal. Considering then that the projection of $\boldsymbol{u}_{\boldsymbol{B}}$ on $\overrightarrow{z_{B}}$ is null from construction of $\mathcal{R}_{B}$, the telescopic assembly constraint is written:

$$
\boldsymbol{u}_{\boldsymbol{B}}={ }^{\boldsymbol{B}} \boldsymbol{R}_{\boldsymbol{i}}\left[\begin{array}{c}
u_{i x} \\
u_{i y} \\
0
\end{array}\right] \quad i=1 \ldots m
$$


where ${ }^{\boldsymbol{B}} \boldsymbol{R}_{\boldsymbol{i}}$ is the rotation matrix describing the rotation of $\mathcal{R}_{i}$ with respect to $\mathcal{R}_{B}$, and where $u_{i x}$ and $u_{i y}$ are the coordinates of $\boldsymbol{u}_{\boldsymbol{i}}$ along $\overrightarrow{x_{i}}$ and $\overrightarrow{y_{i}}$. The $\overrightarrow{z_{i}}$ component of $\boldsymbol{u}_{\boldsymbol{i}}$ corresponds to the twist angular rate $\theta_{i}^{\prime}$, and appears in the curvature projection between $\mathcal{R}_{B}$ and $\mathcal{R}_{i}$ which is written:

$$
\boldsymbol{u}_{\boldsymbol{i}}={ }^{i} \boldsymbol{R}_{\boldsymbol{B}} \boldsymbol{u}_{\boldsymbol{B}}+\theta_{i}^{\prime} \boldsymbol{e}_{\boldsymbol{z}} \quad i=1 \ldots m
$$

where $[\ldots]^{\prime}$ is a derivative according to the arc length $s$ and $\boldsymbol{e}_{\boldsymbol{z}}=\left[\begin{array}{lll}0 & 0 & 1\end{array}\right]^{T}$.

Expressing all the curvature vectors appending in (2) in the same frame $\mathcal{R}_{B}$ using (4), and observing that $\overrightarrow{z_{i}}=\overrightarrow{z_{B}}$ from construction of $\mathcal{R}_{i}$, lead to the following expression of the energy:

$$
E=\frac{1}{2} \int_{s=L_{j}}^{L_{j+1}} \sum_{i=1}^{m}\left(\boldsymbol{u}_{\boldsymbol{B}}+\theta_{i}^{\prime} \boldsymbol{e}_{\boldsymbol{z}}-{ }^{\boldsymbol{B}} \boldsymbol{R}_{\boldsymbol{i}} \hat{\boldsymbol{u}}_{\boldsymbol{i}}\right)^{T} \boldsymbol{K}_{\boldsymbol{i}}\left(\boldsymbol{u}_{\boldsymbol{B}}+\theta_{i}^{\prime} \boldsymbol{e}_{\boldsymbol{z}}-{ }^{\boldsymbol{B}} \boldsymbol{R}_{\boldsymbol{i}} \hat{\boldsymbol{u}_{\boldsymbol{i}}}\right) d s
$$

The potential energy depends thus on the backbone curvature $\boldsymbol{u}_{\boldsymbol{B}}$ and on the twist angles $\theta_{i}, i \in[1, m]$. As explained in [29], these variables are linked by a relation obtained by expressing the balance of moments at each cross section of the robot:

$$
\sum_{i=1}^{m} \boldsymbol{K}_{\boldsymbol{j}} \boldsymbol{u}_{\boldsymbol{B}}=\sum_{i=1}^{m}{ }^{\boldsymbol{B}} \boldsymbol{R}_{\boldsymbol{i}} \boldsymbol{K}_{\boldsymbol{i}}\left(\hat{\boldsymbol{u}}_{\boldsymbol{i}}-\hat{u}_{i z} \boldsymbol{e}_{\boldsymbol{z}}\right)
$$

Thanks to (6), the equilibrium configuration of the robot can be described using the twist angles only. The equilibrium equations can then be obtained by applying the Euler-Lagrange formula to the potential energy (5). We do not consider any assumption on the geometry and mechanical properties of the tubes. The pre-curvature vectors and stiffness matrices are thus arbitrary functions of the arc length $s$. In that case, the Euler-Lagrange formula gives the following equilibrium equations for CTR subject to torsion:

$$
\left\{\begin{array}{l}
k_{i t} \theta_{i}^{\prime \prime}=g_{\theta_{i}}=k_{i t} \hat{u}_{i z}^{\prime}+k_{i t}^{\prime}\left(\hat{u}_{i z}-\theta_{i}^{\prime}\right)-\boldsymbol{u}_{\boldsymbol{B}}^{T} \frac{\partial^{\boldsymbol{B}} \boldsymbol{R}_{\boldsymbol{i}}}{\partial \theta_{i}} \boldsymbol{K}_{\boldsymbol{i}} \hat{\boldsymbol{u}}_{\boldsymbol{i}} \\
i=1 \ldots m
\end{array}\right.
$$

These differential equations are constrained by boundary conditions modeling the proximal actuation of each tube and the free tube extremities. Assuming that the torsional curvature is constant along the transmission lengths as introduced in [7], and that the tubes may have helical initial shapes [8], these conditions are written:

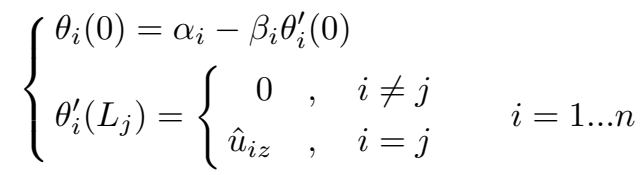

The kinematic model of the CTR is therefore formulated as a so-called twopoint boundary value problem (BVP) composed of the moment balance (6), the 
energetic equilibrium (7) and the boundary conditions (8). For now, this model is section-dependent but can be written along the whole length of the robot by introducing virtual tubes, as described in [14]. The presented technique consists in introducing $n-m$ virtual tubes in a section where $m$ tubes are present, with null bending stiffness in order not to affect real ones. For the same reason, the torsional stiffness is kept constant when extended from a real to a virtual tube since its derivative according to $s$ appends in (7). The stiffness matrix becomes therefore a function of $s$ defined on the total backbone length, i.e. for $s \in\left[0, L_{1}\right]$, and (6)-(7) can be written along the backbone with a constant number $n$ of tubes in interaction. Instead of repeating the BVP for each section, the equilibrium state of the whole CTR can thus be computed with a single set of equations. This will facilitate the implementation of our numerical framework for the analysis of the kinematic model.

\section{Numerical framework}

\subsection{General approach}

We propose in this paper to study numerically the cardinality and the stability of a CTR during its deployment. Our approach is to proceed in four steps, namely the numerical reformulation of the equilibrium determination problem, the deployment simulation, the cardinality assessment and then the stability evaluation. Each of them is here briefly justified before detailed presentation in next section.

The analysis of a CTR is complex since its kinematics are described by a twopoint boundary value problem. As a first step we propose thus to reformulate the equilibrium determination problem in a problem more efficiently solved by numerical tools. Following a classical BVP solving method described in [27], we discretize the robot along its backbone and express the second order derivatives of (7) with finite differences. The CTR is then equivalent to a discrete elastic structure which behavior is described by a set of non-linear equations. We compute equilibrium configuration with dynamic relaxation, successfully used for close problems [25]. The BVP (7) is thus transformed into a classical set of first-order ordinary differential equations (ODE) without considering any assumption on tube number and properties.

CTR are used for their ability to be deployed following complex paths. These paths are, from a geometrical point of view, 1-D manifolds defined in the actuation space. A deployment is thus described by variations of actuation inputs which induce connected sets of equilibria, that we call equilibrium branches according to the vocabulary associated to continuation methods [18]. We indeed propose to rely on such methods to simulate CTR deployment, as they apply to first-order ODE systems, they are not dependent on equilibrium stability and are numerically robust to highly non-linear behaviors as it was illustrated in a close context [20].

In previous works related to CTR analysis, cardinality changes during deployment have been identified considering two kinds of paths: the tubes are 


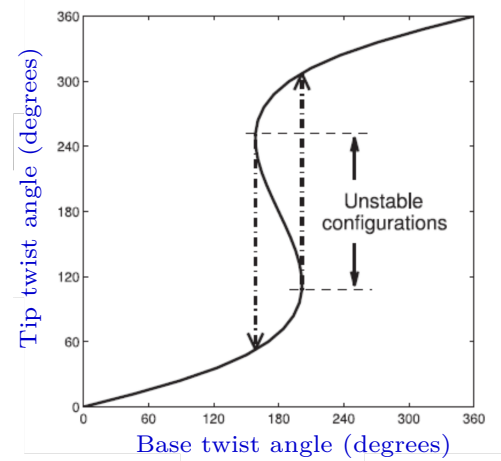

(a) S-curve provided in [14]. Dotted arrows illustrate sudden reconfiguration of the robot between two stable equilibria during the deployment.

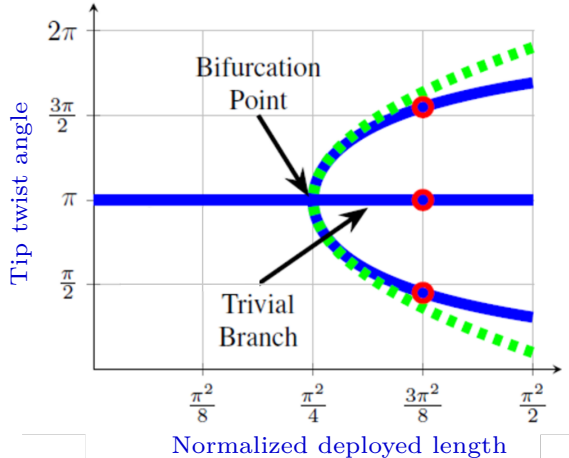

(b) Pitchfork diagram provided in [15]. The green dotted curve corresponds to an approximation of the non-trivial branch obtained with a perturbation method.

Figure 2: Cardinality analysis as performed in the literature.

either rotated relatively to each other $[6,14]$ or placed in opposition and translated simultaneously $[15,7]$. When the robot tip orientation is drawn according to the actuation input, these paths lead to S-shaped curve and pitchfork diagram, as presented on Figure $2 \mathrm{a}$ and $2 \mathrm{~b}$ respectively. Cardinality changes are spotted along these diagrams by particular equilibria located at points with infinite tangent slope and at branch intersection. These equilibria correspond indeed exactly to the mathematical entities designated as Limit Point bifurcations (LP) and Branch Point bifurcations (BP) respectively, as introduced and studied in the bifurcation theory $[19,18]$. We propose then to use the existing knowledge on these kinds of bifurcations to detect cardinality changes. The bifurcation theory provides especially generic estimators of the presence of bifurcation which, when coupled with the equilibrium branch computation, gives a numerical method for robot cardinality assessment without having to consider any assumption on the tubes.

The equilibrium determination problem after reformulation is close to the one addressed by Lazarus et al. in the context of elastic buckled beams [20]. The discretization process allows them to establish a simple stability criteria based on the Jacobian matrix of a discrete beam kinematic model. We propose thus to follow their approach in order to obtain a generic numerical method for the CTR stability evaluation which fits with the continuation and bifurcation tools.

Overall, the proposed reformulation stage and numerical methods form a numerical framework for the cardinality and stability analysis of deploying CTR. In the following, each of the above-mentioned stages is now detailed in order to clarify it and make the framework easily reproducible. 


\subsection{Reformulation of the equilibrium determination problem}

Boundary value problems are classically solved using a finite difference method as explained in [27]. Here, such method is being used after first discretizing the CTR in a number $N$ of points between $s=0$ and $s=L_{1}$ as denoted for illustration on Figure 3, a point $k$ being located at the arc length $s_{k}$. The number of points for section $j$ is designated by $N_{j}$ so that $N=\sum_{j=1}^{n} N_{j}$. The twist angles defining the equilibrium configuration of the robot are then evaluated at each point, as illustrated on the figure for point 5 and point $N_{3}+3$. The distance in arc length between point $k$ and point $k+1$ is denoted $h_{k}$, so that $h_{k}=s_{k+1}-s_{k}$.

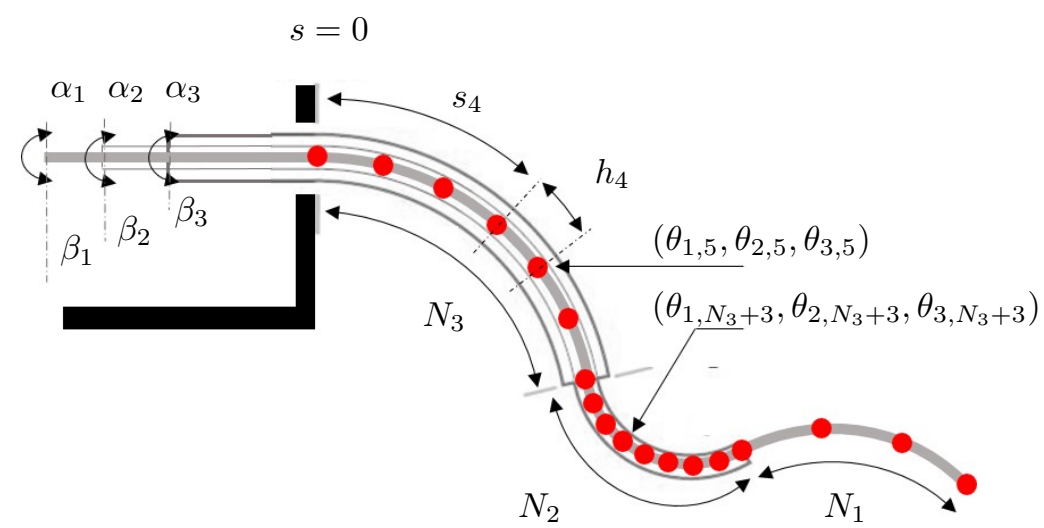

Figure 3: Representation of the CTR after discretization. Here $n=3$ and $\left[\begin{array}{lll}N_{1} & N_{2} & N_{3}\end{array}\right]=$ $\left[\begin{array}{lll}4 & 8 & 6\end{array}\right]$.

The equilibrium equation (7) can then be written using discrete formulation of derivatives. The second-order derivative of $\theta_{i}(s)$ is replaced by a central second-order finite difference, so that (7) becomes:

$$
k_{i t, k} \frac{h_{k} \theta_{i, k-1}-\left(h_{k}+h_{k-1}\right) \theta_{i, k}+h_{k} \theta_{i, k-1}}{h_{k} h_{k-1}^{2}}-g_{\theta_{i}, k}=0
$$

with $i=1 \ldots n, k=2 \ldots N-1$ and subscript $k$ denoting an evaluation at point $k$. The initial and final boundary conditions (8) are then included to the central finite differences evaluated at robot extremities:

$$
\begin{aligned}
& k_{i t, 1} \frac{\beta_{i} \theta_{i, 2}-\left(\beta_{i}+h_{1}\right) \theta_{i, 1}+h_{1} \alpha_{i}}{h_{1} \beta_{i}^{2}}-g_{\theta_{i}, 1}=0 \\
& k_{i t, N} \frac{\theta_{i, N f}-2 \theta_{i, N}+\theta_{i, N-1}}{h_{N}^{2}}-g_{\theta_{i}, N}=0 \\
& \theta_{i, N f}=\hat{u}_{i z, N} h_{N}+\theta_{i, N} \\
& i=1 \ldots n
\end{aligned}
$$

Gathering the twist angles in a state vector $\boldsymbol{X}$ such that:

$$
\boldsymbol{X}=\left[\begin{array}{llllll}
\theta_{1,1} & \ldots & \theta_{1, N} & \theta_{2,1} & \ldots & \theta_{n, N}
\end{array}\right]^{T},
$$


and considering a fixed set of actuation inputs, the boundary-value problem is thus transformed into a set of non-linear equations of the form:

$$
G(X)=0
$$

Such formulation of CTR kinematic model is similar to the one involved in the shape finding of elastic structures such as tensegrity mechanisms [25] and fabrics [28]. Dynamic relaxation was successfully used for these classes of systems to solve the so-called form-finding problem. We therefore propose to use dynamic relaxation, where state variables are artificially considered functions of time. The robot is initially constrained in an arbitrary shape and then relaxed virtually until it reaches an equilibrium. The virtual dynamics of the state variables according to the virtual time are imposed to fit a second order damped differential equation:

$$
M \ddot{\boldsymbol{X}}+D \dot{\boldsymbol{X}}+\boldsymbol{G}(\boldsymbol{X})=\mathbf{0}
$$

with $M$ and $D$ two constant positive scalars in order to ensure numerical stability [25]. Selection of these values will be discussed later.

One can easily check that the steady state of (13) is a solution of (12). System (13) can be also written as a set of first-order ordinary differential equations (ODE), that is more efficient numerically to solve using the state-space vector $\boldsymbol{Y}=\left[\begin{array}{ll}\boldsymbol{X}^{T} & \dot{\boldsymbol{X}}^{T}\end{array}\right]^{T}$. The equilibrium determination problem consists then in solving a set of $2 \times n \times N$ first-order ODE of the form:

$$
\dot{\boldsymbol{Y}}=\boldsymbol{A}(\boldsymbol{Y})
$$

with

$$
\boldsymbol{A}(\boldsymbol{Y})=\left[\begin{array}{cc}
\mathbb{O} & \mathbb{I} \\
\mathbb{O} & -\frac{1}{M} D \mathbb{I}
\end{array}\right] \boldsymbol{Y}+\left[\begin{array}{c}
\mathbf{0} \\
-\frac{1}{M} \boldsymbol{G}(\boldsymbol{X})
\end{array}\right]
$$

and where $\mathbb{O}$ and $\mathbb{I}$ are respectively null matrices and identity matrices of size $n N$. The steady state of this system, that we call equilibrium of (14) in the following, is denoted $\boldsymbol{Y}^{\star}$.

\subsection{Deployment simulation through continuation method}

As described earlier, CTR deployment is considered described in actuation space. We therefore have prescribed variations of $L_{i}$ and $\alpha_{i}, i=1 \ldots n$, and we wish to compute branches of equilibria as the robot is deployed along a path using a continuation method. Two major types of continuation methods can be applied in that case: the Predictor Corrector Method (PCM) [18] and the Asymptotic Numerical Method (ANM) [24]. The ANM can be an efficient tool in terms of accuracy and computation time if the equilibrium equations are written in a quadratic form. This pre-processing step is tricky since it is not generic. As shown for elastic structures in [20] and [2], different intermediate variables and casting techniques must be introduced depending on the considered geometric parametrization and constitutive equations. We therefore propose to use PCM since it does not require any specific pre-processing. Its relevance will be further discussed in terms of accuracy and computation time in Section 4. 
In order to describe the PCM principle, let us consider the situation where the $i$-th tube of a CTR is deployed by performing a translation. This corresponds to a modification of the actuation input $L_{i}$, which becomes an input variable of the system (15). The modification of $L_{i}$ lead then to the computation of a branch $\mathcal{B}$ of equilibria $\boldsymbol{Y}^{\star}$ defined by:

$$
\begin{aligned}
\mathcal{B}: \mathbb{R} & \rightarrow \mathbb{R}^{2 n N} \\
L_{i} & \rightarrow\left(\boldsymbol{Y}^{\star} \mid \boldsymbol{A}\left(\boldsymbol{Y}^{\star}, L_{i}\right)=\mathbf{0}\right)
\end{aligned}
$$

The PCM provides a discrete representation of the branch $\mathcal{B}$ with a number $N_{p}$ of points. These points are computed iteratively by a two-step process composed of a prediction step followed by a correction step. To describe them, let us note $\left(L_{i, k-1}, \boldsymbol{Y}_{k-1}^{\star}\right)$ the current equilibrium. In a first step, the length $L_{i, k-1}$ is incremented by the so-called continuation step $\delta_{c}$. The equilibrium corresponding to this actuation increment is predicted following the branch tangent according to $L_{i}$, so that:

$$
\left\{\begin{aligned}
L^{P} & =L_{i, k-1}+\delta_{c} \\
\boldsymbol{Y}^{P} & =\boldsymbol{Y}_{k-1}^{\star}+\delta_{c} \frac{\partial \mathcal{B}}{\partial L_{i}}\left(L_{i, k-1}\right)
\end{aligned}\right.
$$

In a second step, the predicted point $\left(L_{i}^{P}, \boldsymbol{Y}^{P}\right)$ is corrected until the new equilibrium point of the branch $\left(L_{i, k}, \boldsymbol{Y}_{k}^{\star}\right)$ is accurately found. Following [18], this step is performed by applying a Gauss-Newton algorithm, starting from $\left[\begin{array}{ll}\boldsymbol{Y}^{P} & L_{i}^{P}\end{array}\right]$, to the system of equations:

$$
\left\{\begin{array}{l}
\boldsymbol{A}\left(\boldsymbol{Y}, L_{i}\right)=\mathbf{0} \\
\left\langle\boldsymbol{Y}-\boldsymbol{Y}^{P}, \frac{\partial \mathcal{B}}{\partial L_{i}}\left(L_{i, k-1}\right)\right\rangle=0
\end{array}\right.
$$

where the operator $\langle$,$\rangle denotes the dot product operator.$

These two steps require the considered system of equation to be smooth and to have a full rank Jacobian matrix. The first condition is verified since the discrete CTR kinematic model solved with dynamic relaxation (14) is a composition of smooth functions. In cases where the Jacobian matrix becomes singular, the authors in [18] provide alternative root finding methods for the correction step so that the PCM does not fail.

Because of the non-linear phenomena involved in the behavior of CTR, the curvature of an equilibrium branch can vary during its computation. This can impact the quality of the prediction step and on the numerical stability of the Gauss-Newton algorithm. The PCM is therefore used with a step size control algorithm described in [18], which automatically reduces $\delta_{c}$ in case of numerical stability loss. Otherwise, the step size is increased until the user-defined maximum value is reached in order to optimize the length of the computed branch.

One very interesting feature of the method is that it does not assume the robot to be stable. The continuation tool can thus be used to compute any 
branch of equilibria, including unstable configurations. This allows in particular to find stable branches in continuation of unstable ones, which would have hardly been computed with a standard numerical tool depending on initial conditions. This property will be outlined in the following examples. To sum up the advantages of PCM in our context, the use of a step size control algorithm and of alternative correction methods with the PCM provides an efficient tool for CTR deployment simulation which is numerically robust to highly non-linear behaviors, to changes in robot stability and to kinematic model singularities.

\subsection{Cardinality analysis}

Our approach to assess cardinality is to detect LP and BP bifurcations along branches of equilibria, as introduced in Section 3.1. The term "bifurcation" has been indeed used for CTR previously, in [15] and [7], to describe branch intersections. We extend here the use of bifurcation notion, defined in the following as changes in the robot behavior along a branch, as stated in [18], and we consider the associated classification.

Existing estimators for bifurcation detection, designated as locators, are being used. They are mathematical functions which become zero with a sign change when a bifurcation is encountered. The locators of LP ans BP bifurcations as introduced in $[19,18]$ write in our context:

$$
\begin{gathered}
\phi_{L P}=\frac{\partial \mathcal{B}}{\partial L_{i}} \\
\phi_{B P}=\operatorname{det}\left(\left[\begin{array}{cc}
\boldsymbol{A}_{\boldsymbol{Y}} & \frac{\partial \boldsymbol{A}}{\partial L_{i}} \\
\frac{\partial \mathcal{B}}{\partial \boldsymbol{Y}} & \frac{\partial \mathcal{B}}{\partial L_{i}}
\end{array}\right]\right)
\end{gathered}
$$

where $\boldsymbol{A}_{\boldsymbol{Y}}$ is the Jacobian matrix of $\boldsymbol{A}(\boldsymbol{Y})$ with respect to $\boldsymbol{Y}$.

The detection and the location of cardinality changes is then integrated to the continuation process following the method given in [18]. Locators are evaluated for each equilibrium configuration computed with the PCM. A sign change of one locator between two points of the branch indicates the existence of the corresponding bifurcation. The latter is then located accurately between the two points with a root finding method applied on:

$$
\left\{\begin{array}{l}
\boldsymbol{A}(\boldsymbol{Y})=\mathbf{0} \\
\phi_{B P}=0 \text { or } \phi_{L P}=0
\end{array}\right.
$$

The Jacobian of (21) may be singular in the neighbourhood of the bifurcation. This is typically the case at BP bifurcation, where the vanishing of (20) may be due to a singular $\boldsymbol{A}_{\boldsymbol{Y}}$. This hampers the use of a standard Gauss-Newton method for the localization. A secant method is instead preferred, since it does not require the inversion of $\boldsymbol{A}_{\boldsymbol{Y}}$ [27] and it has been successfully used in [18] to locate bifurcations.

To sum up the advantages of our approach for cardinality assessment, the PCM and the bifurcation theory are employed here with a generic kinematic 
model of CTR, solved with dynamic relaxation. We construct then a graph composed of computed equilibrium branches and related bifurcations, known as bifurcation diagram, through which the CTR cardinality is assessed during a deployment. The association of equilibrium branch determination with detection of bifurcation provides thus a numerical method which is valid for any tube number and properties. This genericity has never been offered previously to the best of our knowledge.

\subsection{Local stability assessment}

Our second goal is to evaluate the local stability of a CTR during its deployment. The equilibrium determination problem after reformulation is close to the one addressed by Lazarus et al. in the context of elastic buckled beams [20]. The stability of a loaded beam is then assessed by verifying that its equilibrium configuration corresponds to a potential energy minimum, i.e. that the Hessian matrix of the potential energy is positive definite. The beam discretization that is then initially performed allows the authors to build such a Hessian matrix based on the Jacobian matrix of the energy-based equilibrium equations, leading to a simple numerical stability criteria.

We use this approach in order to establish a numerical method able to assess CTR stability and which fits the continuation and bifurcation tools. After the reformulation stages, the robot potential energy depends only on the discrete and independent variables gathered in the state vector $\boldsymbol{X}$ described in (11). An equilibrium state $\boldsymbol{X}^{\star}$ solution of (12) is thus stable if the Hessian matrix of the potential energy with respect to $\boldsymbol{X}$ is positive definite when evaluated at $\boldsymbol{X}^{\star}$. The equilibrium equations (7) are derived directly from the energy expression in section 2. The Hessian matrix corresponds therefore simply to the Jacobian matrix of $\boldsymbol{G}$ with respect to $\boldsymbol{X}$, denoted $\boldsymbol{G}_{\boldsymbol{X}}$. An equilibrium state $\boldsymbol{X}^{\star}$ is consequently stable if $\boldsymbol{G}_{\boldsymbol{X}}$ is defined positive, which is written:

$$
\boldsymbol{G}_{\boldsymbol{X}}\left(\boldsymbol{X}^{\star}\right) \succ 0
$$

In the opposite state the equilibrium is unstable if $\boldsymbol{G}_{\boldsymbol{X}}$ has at least one strictly negative eigenvalue.

In terms of implementation, we use the numerical evaluation of (22) to assess the CTR stability. This method needs the computation of $\boldsymbol{G}_{\boldsymbol{X}}$ and its eigenvalues. We can demonstrate easily from (14) that the former one is included into the Jacobian matrix $\boldsymbol{A}_{\boldsymbol{Y}}$, which writes:

$$
\boldsymbol{A}_{\boldsymbol{Y}}=\left[\begin{array}{cc}
\mathbb{O} & \mathbb{I} \\
-\frac{1}{M} \boldsymbol{G}_{\boldsymbol{X}} & -\frac{D}{M} \mathbb{I}
\end{array}\right]
$$

The determination of $\boldsymbol{G}_{\boldsymbol{X}}$ is then straightforward since $\boldsymbol{A}_{\boldsymbol{Y}}$ is already computed in the correction step of the PCM and in the locator of BP bifurcations. We use then the QR decomposition algorithm to obtain the eigenvalues as $\boldsymbol{G}_{\boldsymbol{X}}$ is square and non symmetric. Since $\boldsymbol{G}_{\boldsymbol{X}}$ is computed from a generic kinematic model of CTR, this numerical method is valid for any number, geometry and mechanical properties of the tubes. 
3.6 Implementation

\subsection{Implementation}

The previous sections showed that the cardinality and stability analysis of a CTR during its deployment results from continuation method and bifurcation analysis applied on discretized model of CTR initially solved with dynamic relaxation method. These model reformulation stages and numerical methods constitute our numerical framework which is implemented following the flow chart diagram presented on Figure 4. We now give details of implementation corresponding to the three blocks composing the diagram.

The continuation method and bifurcation analysis are available as off-theshelf tools using the software AUTO [5] or the Matlab toolbox CL-MatCont [4]. Both tools use PCM and step size control algorithms for equilibrium branch computation and provide locators for the detection of limit points (LP) and branch points (BP). CL-MatCont provides the most advanced bifurcation analysis for dynamical systems as explained in [12]. It allows moreover to define personalized test functions evaluated during the continuation process which can be used to integrate the local stability criteria defined in (22). Consequently, CL-MatCont suits totally to our needs in term of deployment simulation, bifurcation analysis and stability evaluation, and its use is designated as a single dashed-dotted block on Figure 4.

The deployment simulation requires then the user to perform two programming steps using the functions of the Matlab toolbox, surrounded with a solid line on the flow chart. First, the discrete model of CTR formulated as an ODE system (14) must be written in a Matlab file called System. This file must be filled also with initialization and user-defined test functions which compute the initial equilibrium configuration of the robot through dynamic relaxation and the local stability criteria (22) respectively. Second, a simulation program must be coded with the toolboxes functions in order to call the initialization functions, to specify the path to perform and to set continuation settings. An example of such a simulation program is given in Appendix A. The execution of this script results thanks to CL-MatCont in a set of variables which contains the computed branches of equilibria, the stability of the corresponding equilibrium configurations and the nature and the location of the detected bifurcations.

As a summary, our proposition of numerical framework for the cardinality and stability assessment of a CTR during its deployment, summed up in Figure 4, requires few implementation efforts from the user. The reformulation of the equilibrium determination problem (Discretization, Finite difference, Dynamic relaxation) is performed in this paper for a generic kinematic model of robot and can be consequently re-used. The equilibrium branches computation (Step size control, Prediction, Correction) and the bifurcation detection (Detection/Location of bifurcation) are already implemented in- and performed automatically by CL-MatCont. The deployment simulation, the cardinality assessment and the stability evaluation require then the user to follow only two steps composed of simple Matlab programming (MatCont System file, Simulation program).

This fully numerical and generic approach for CTR behavior analysis has never been proposed to the best of our knowledge. It contrasts with the current 


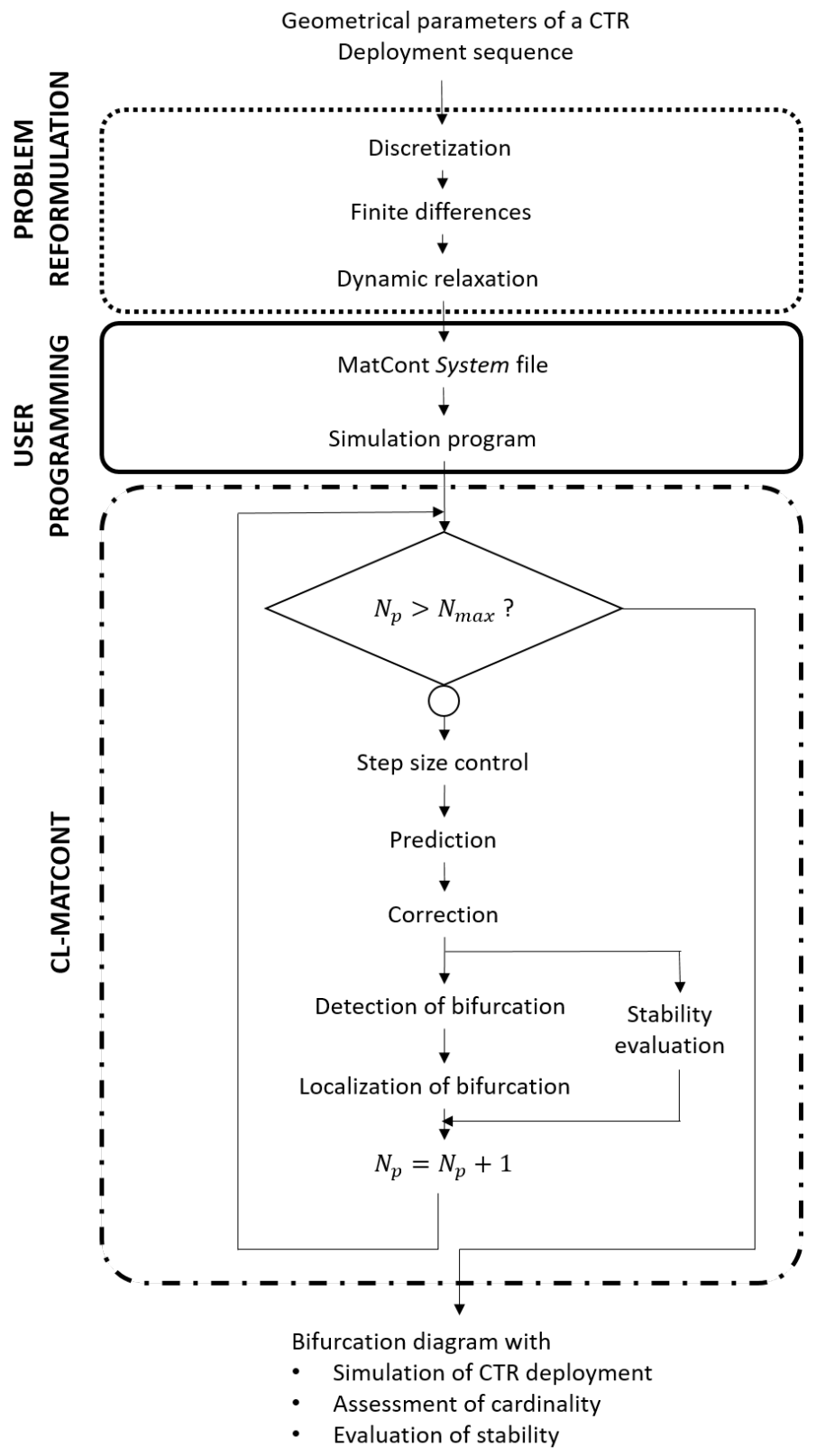

Figure 4: Flow chart diagram representing the proposed numerical framework. Steps developed in this paper are surrounded with a dashed line. Steps which must be implemented by the user are surrounded with a solid line. The steps performed automatically by MatCont are surrounded with a dash-dotted line. 


\begin{tabular}{|c|c|c|}
\hline Parameter & Tube 1 & Tube 2 \\
\hline$k_{f i}(M P a)$ & 1 & 1 \\
$k_{t i}(M P a)$ & $1 / 1.3$ & $1 / 1.3$ \\
$\hat{u}_{i x}\left(\mathrm{~mm}^{-1}\right)$ & $1 / 60$ & $1 / 60$ \\
& & \\
\hline
\end{tabular}

Table 1: Design parameters of the two-tube CTR for case study.

\begin{tabular}{|c|c|}
\hline Parameter & Value \\
\hline$D$ & -10 \\
$M$ & -1 \\
$N_{p}$ & 100 \\
MaxStepSize & 10 \\
\hline
\end{tabular}

Table 2: Parameters for numerical evaluation of the CTR.

works which attempt to perform these analyses analytically at the cost of model simplifications. The capabilities of our numerical framework are demonstrated in two steps in the rest of this paper: reference cases of CTR deployment are first studied in order to validate the approach and to evaluate its performances. Second, new case studies are investigated, leading to new results of interest for CTR exploitation.

\section{Method assessment with a two-tube CTR}

A well-known situation with CTR cardinality and stability issues depicts a robot composed of two tubes fully overlapped, with constant stiffness and planar pre-curvature, which is deployed in free space. This case study has been extensively considered because of the kinematic model simplicity and its correlation with physical prototypes. Consequently, we propose here to focus on this robot configuration and simulate the two deployments described in section 3.1. This will allow us to reproduce two standard results of two-tube CTR: the critical interaction length $[15,7]$ and the "S-curve" $[6,14]$ describing the robot tip orientation according to its actuation angles.

\subsection{Critical interaction length}

Hendrick et al. established a global stability criterion assuring, if respected, that the CTR cannot snap for any rotation of the tubes [15]. This criterion imposes conditions on the interaction length between the tubes, denoted $\Delta L_{2}$ according to the notations introduced in Section 2, the transmission length, the stiffness and the pre-curvatures of the tubes so that:

$$
\frac{-\cot (\lambda)}{\sqrt{\lambda}}<\beta_{\sigma}
$$

with

$$
\lambda=\Delta L_{2}^{2} \hat{u}_{1 x} \hat{u}_{2 x} \frac{k_{1 b} k_{2 b}\left(k_{1 t}+k_{2 t}\right)}{k_{1 t} k_{2 t}\left(k_{1 b}+k_{2 b}\right)}
$$

where $\beta_{\sigma}$ is the normalized transmission length defined by:

$$
\beta_{\sigma}=\frac{\beta_{1} k_{2 t}+\beta_{2} k_{1 t}}{\Delta L_{2}\left(k_{1 t}+k_{2 t}\right)}
$$


4.1 Critical interaction length

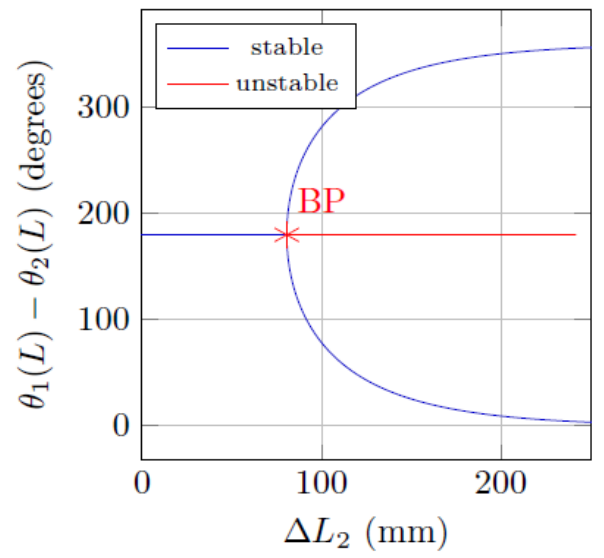

(a) Pitchfork diagram obtained with the numerical framework for $\beta_{\sigma}=0$.

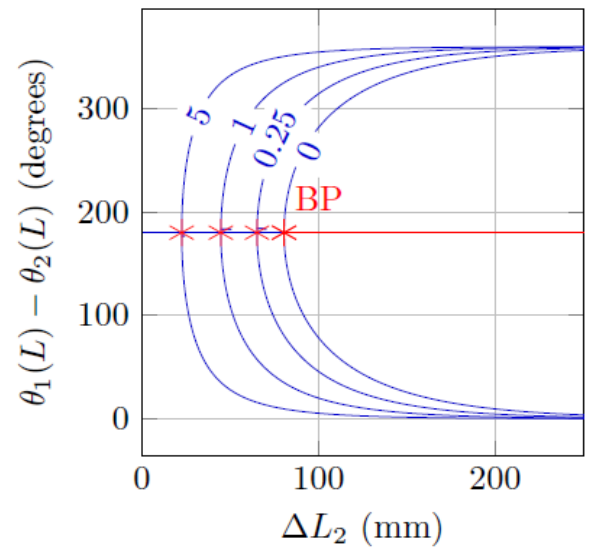

(b) Evolution of the pitchfork diagram according to several values of $\beta_{\sigma}$. Values of transmission length are written in blue.

Figure 5: Bifurcation diagram according to the variation of $\Delta L_{2}$.

If the initial shape and the mechanical properties of the tubes are set, equation (24) suggests that there is a critical value for $\Delta L_{2}$, denoted $\Delta L_{2 c}$ in the following, beyond which the CTR is unstable. This critical interaction length has also been highlighted in the pitchfork diagrams of Hendrick et al. [15] represented in Figure 2b, where it is located at the two branches intersection.

We propose to reproduce the pitchfork diagram with our numerical framework. The considered geometry and mechanical properties of the tubes are gathered in Table 1. The kinematic model solved with dynamic relaxation (14) is written for $n=2$ in a CL-MatCont System file. We consider equal transmission lengths for the two tubes, an idealization used in [15] in order to observe the evolution of the pitchfork diagram according to $\beta_{\sigma}$. The value of the transmission length is then computed using (26). All the non-mentioned parameters are assumed to equal zero. The virtual damping and inertia given in Table 2 are chosen so that the robot initial equilibrium configuration can be computed by integrating (14) with the standard Matlab ode45 solver. Experience shows that any negative values of $M$ and $D$ lead to a numerically stable dynamical system. Their magnitude seems to have few influence on the numerical stability. We set then the damping ten times higher than the inertia since it provides an appropriate time response, in terms of oscillations and convergence speed, a remark that is valid for all the simulations presented in the following.

The path composed of the simultaneous translation of the two tubes is parametrized by $\Delta L_{2}$. We write thus a simulation program which specifies this section length as the parameter to be varied during the simulation by continuation. The script initializes the CTR in a configuration where tubes are in opposition following [15], i.e. $\left(\alpha_{1}, \alpha_{2}\right)=(0, \pi)$, and where $\Delta L_{2}$ is chosen close to 0 . The discretized model of CTR does not allow null section lengths since finite differences are divided by $h_{k}$. The settings of the PCM and of the 
step size controller used for the deployment simulation are specified in Table 2 . We choose the number of point $N_{p}$ and the maximum step size by trial and error process so that the two tubes are deployed towards $\Delta L_{2}=250 \mathrm{~mm}$. The non-specified settings are left to their default values as given in [4].

Starting from the initial configuration at $\Delta L_{2} \approx 0$, the computed branch is stable (plotted in blue) until it reaches a BP bifurcation. The branch becomes then unstable (plotted in red). Starting from the detected branch point, it is possible thanks to CL-MatCont to draw the second intersecting branch. Beyond the bifurcation, the CTR can thus have two stable and one unstable equilibrium configurations for the same interaction length as illustrated on the complete pitchfork diagram in Figure 5a. This bifurcation diagram can be easily computed for several values of $\beta_{\sigma}$, resulting in a set of graphs superposed in Figure $5 \mathrm{~b}$. The evolution of equilibrium branches according to the interaction and transmission lengths is identical as presented in [15].

The length at the BP bifurcation corresponds to a numerical estimation of the critical interaction length. Since the accuracy of the discrete derivatives used in (9) depends on the sample number along the section $N_{2}$, so does the accuracy of the computed branches and of the bifurcation location. The accuracy is expected to increase with $N_{2}$, as well as the computation time since continuation and bifurcation tools need to compute and inverse the $2 n\left(N_{1}+N_{2}\right) \times 2 n\left(N_{1}+N_{2}\right)$ Jacobian matrix $\boldsymbol{A}_{\boldsymbol{Y}}$. The numerical estimation of the critical length is therefore compared to the theoretical value given by (24) for different number of points in the case where $\beta_{\sigma}=0$. The relative error equals $2.45 \%$ when $N_{2}=20$, which is acceptable as quick evaluation, and drops to $0.03 \%$ when $N_{2}=100$. Computation time increases as expected from 20s to 370s respectively, keeping however reasonable values. We observe furthermore that choosing $N_{2}=20$ does not impact the result of the cardinality and stability evaluation. This number of point is consequently chosen for the next simulations in order to optimize accuracy and computation time.

\subsection{Tip rotation versus actuation angles}

As described in section 3.1, the S-shaped curve is the equilibrium branch corresponding to the deployment scenario where one of the tubes is rotated with respect to the other. In order to simulate this deployment, we set $\alpha_{2}=0$ and define $\alpha_{1}$ as the parameter to be varied during the PCM. We consider the same CTR, which parameters are gathered in Table 1. The interaction length $\Delta L_{2}$ is set to $100 \mathrm{~mm}$, beyond the critical length obtained previously. We consider the continuation parameters gathered in Table 2 .

The resulting bifurcation diagram is presented on Figure 6a. Starting from the initial configuration where $\alpha_{1}=0$, a stable branch is computed until a LP bifurcation. The next equilibria are unstable and stability is obtained again after passing through a second LP. The CTR can thus have three different equilibria for the same actuation inputs, two stables and one unstable. The local stability evaluation is consequently validated, since it corresponds to the evaluation presented on the different S-shaped curves in the literature like the curve illustrated in Figure 2a. 


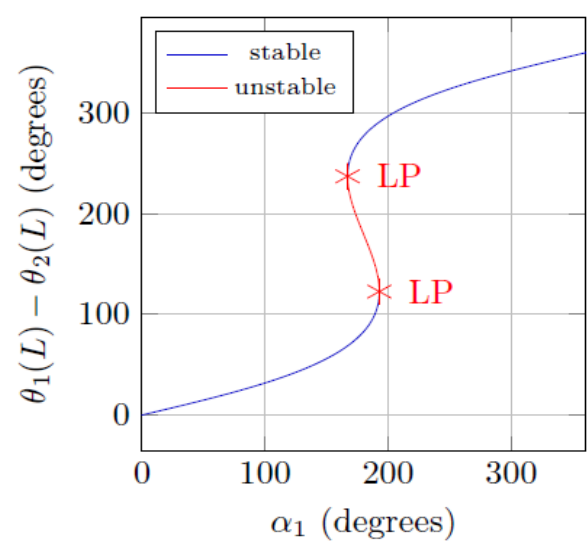

(a) S-shaped curve obtained with the numerical framework. Stable configurations in blue. Unstable configurations in red.

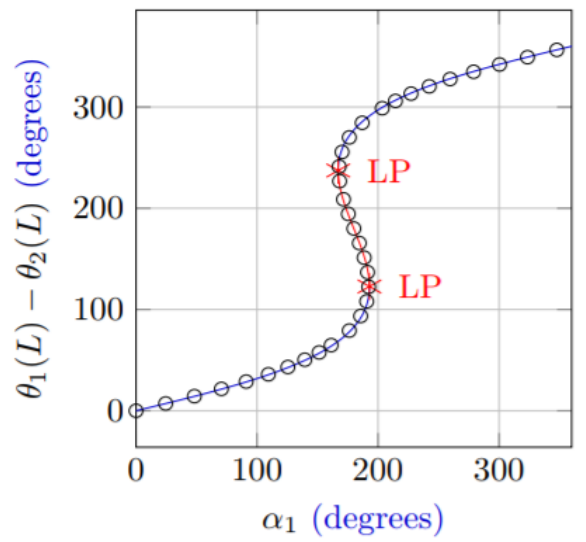

(b) Comparison of the framework results with the backward IVP method (black circles).

Figure 6: Simulation of relative rotation of the tubes for a two-tube robot.

In order to validate these results with respect to the S-shaped curves plotted in the literature, the same diagram is generated with the backward initial value problem (IVP) method used in [13] and [14] and applied to (7). Figure 6b shows the correlation between the two curves for $N_{2}=100$, where the relative error is $0.9 \%$.

This case study illustrates the capability of the proposed framework to compute equilibrium branches without being dependent on the CTR stability. The second stable branch is obtained automatically in the continuation of the unstable one, without needing a priori knowledge on it.

To summarize the presented analysis of the two-tube CTR reference cases, the proposed numerical framework allows us first to determine the static behavior of the robot during a deployment. Second, it provides the local stability of the computed equilibria and critical values of parameters for global stability. Third, it allows to assess the CTR cardinality correctly. Numerical errors can be reduced to admissible values by increasing the number of discretization points along the robot. This gain in term of accuracy implies reasonable computation times, validating thus the interest of the proposed numerical approach.

\section{Method exploitation for a three-tube CTR}

As outlined in the introduction, the stability analysis of CTR with more than two tubes is limited in the literature to local and global stability criteria if the tubes do not have torsion pre-curvature, and to local stability criteria only when arbitrary shapes are considered. There is in particular no information about the cardinality of robots deploying in a FTL manner with tubes in opposition and 
about the critical length of robots with helical pre-curvature. This last case is of importance since it allows for FTL deployment with a larger variety of tube initial shapes [8]. The proposed numerical approach is therefore used to bring new missing information on such geometries. In order to use realistic numerical values for CTR geometry, we use a medical scenario developed in [10] of olfactory cell exploration.

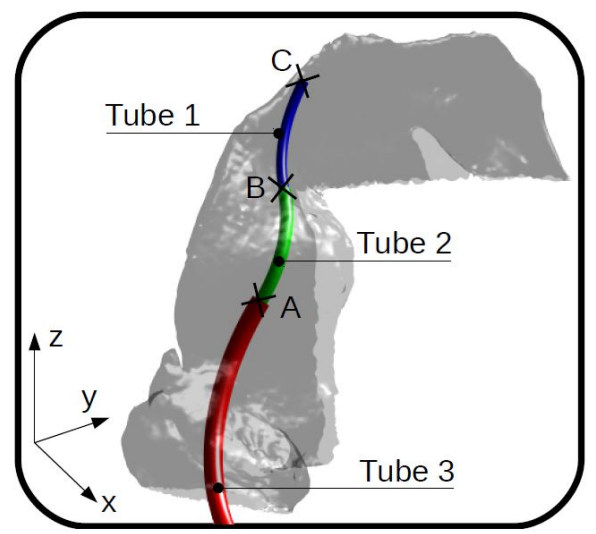

Figure 7: CTR after FTL deployment of three section paths as described in [10].

\begin{tabular}{|c|c|c|c|}
\hline & Tube 1 & Tube 2 & Tube 3 \\
\hline$k_{f i}(\mathrm{MPa})$ & 1.51 & 2.64 & 13.48 \\
$k_{t i}(\mathrm{MPa})$ & 4.34 & 7.58 & 38.75 \\
$\hat{u}_{i x}\left(\mathrm{~mm}^{-1}\right)$ & 0.059 & 0.136 & 0.066 \\
$\alpha_{i}(\mathrm{rad})$ & 0 & $\pi$ & 0 \\
\hline
\end{tabular}

Table 3: Kinematic model parameters used in the study of the three-tube CTR.

\begin{tabular}{|c|c|c|c|c|}
\hline \multicolumn{3}{|c|}{ Actuated tubes } & \multicolumn{2}{c|}{ Section length $\left(\Delta L_{1}, \Delta L_{2}, \Delta L_{3}\right)$} \\
\hline Tube 1 & Tube 2 & Tube 3 & Initial value & Final value \\
\hline$\checkmark$ & $\checkmark$ & $\checkmark$ & $(0,0,0)$ & $\left(0,0, \Delta L_{3 f}\right)$ \\
$\checkmark$ & $\checkmark$ & $\times$ & $\left(0,0, \Delta L_{3 f}\right)$ & $\left(0, \Delta L_{2 f}, \Delta L_{3 f}\right)$ \\
$\checkmark$ & $\times$ & $\times$ & $\left(0, \Delta L_{2 f}, \Delta L_{3 f}\right)$ & $\left(\Delta L_{1 f}, \Delta L_{2 f}, \Delta L_{3 f}\right)$ \\
\hline
\end{tabular}

Table 4: FTL deployment sequence of a three-tube CTR. 


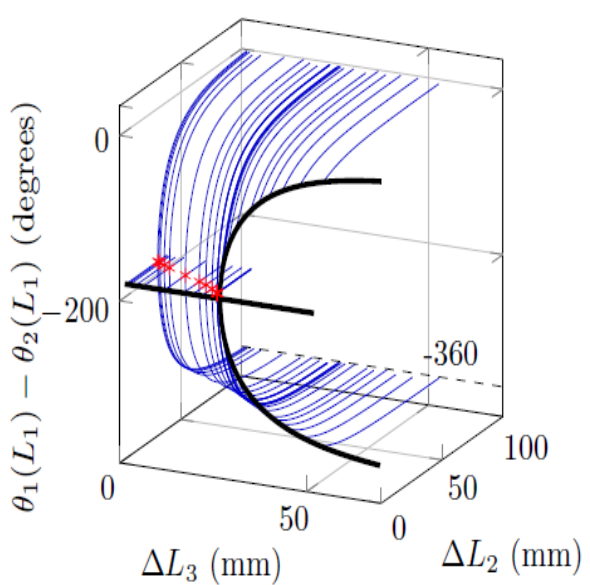

(a) Isometric view

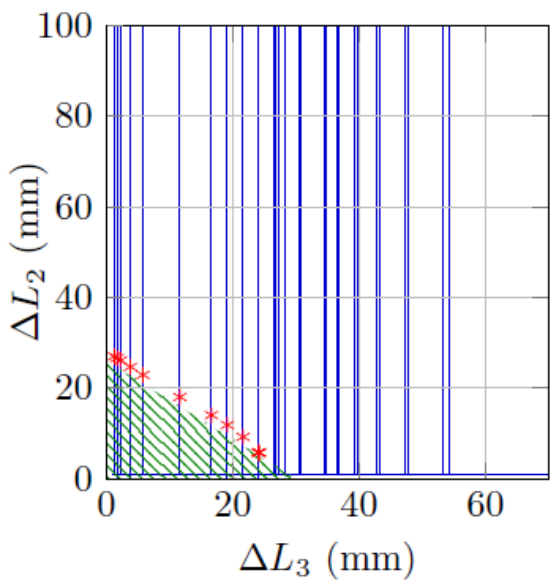

(b) $\left(\Delta L_{3}, \Delta L_{2}\right)$ plane

Figure 8: Equilibrium branches during the FTL deployment of a three-tube CTR. The tip torsion angle is represented as a function of the two actuated lengths. BP bifurcations are plotted in red.

\subsection{Cardinality during FTL deployment}

The considered CTR is composed of three tubes, with planar and constant pre-curvature, constant stiffness and mounted in opposition. The corresponding model parameters are gathered in Table 3 . The robot must deploy with strong geometrical constraints due to the narrowness of nasal cavities as shown on Figure 7. For that purpose, FTL deployment is considered with obvious need for stability during deployment.

To achieve a FTL deployment, the tubes must be actuated in a specific sequence in addition of the geometrical constraints. This sequence consists in deploying the sections introduced in Section 2, i.e. translating simultaneously several tubes, in the descending order. The followed trajectory can therefore be parametrized with section lengths only, which must reach reference values denoted $\Delta L_{i f}$ for section $i$. In the case study considered here, the deployment sequence is composed of three steps which are described in Table 4.

We only consider the first two steps since they correspond to situations with variable lengths of interaction, which can cause cardinality changes and instabilities. In order to follow the deployment sequence, a first bifurcation diagram according to $\Delta L_{3}$ is computed following the proposed numerical framework. Successive continuations according to $\Delta L_{2}$ are then performed starting from several equilibrium configurations of this diagram. This corresponds to start from several values of $\Delta L_{3 f}$ and to deploy the second section. We use the dynamic relaxation parameters and the continuation settings of the previous validation studies (Tables 2).

The resulting graph is presented on Figure 8a, where the stable and unstable 
branches are not distinguished for the sake of readability. The first continuation according to $\Delta L_{3}$ reveals a $\mathrm{BP}$ bifurcation, indicating that a second branch intersects. Computing this branch leads to a pitchfork bifurcation diagram plotted in black. The section length at the BP can thus be considered as a critical interaction length between the three tubes of section 3, called $\Delta L_{3 c}$. The successive continuation steps according to $\Delta L_{2}$ reveal also BP bifurcations, which are marked on the graph with red stars.

This set of bifurcation diagrams are represented in a 3D graph, proposed for the first time here, which is easily produced with our numerical framework and which gives three kinds of information. First, during the considered FTL deployments, the CTR can have two stable and one unstable equilibrium states for the same actuation inputs. Cardinality changes appear during the second deployment step only in the case where $\Delta L_{3 f}<\Delta L_{3 c}$. Once the robot has bifurcated, the tubes are no more placed in opposition and tend to align as $\Delta L_{3}$ or $\Delta L_{2}$ increases, i.e. the angle difference between the tubes converge toward 0 degrees or -360 degrees. Second, the critical length of section 2, denoted $\Delta L_{2 c}$, depends on $\Delta L_{3 f}$ with a quasi-linear relation observed from the graph top view in Figure 8b. Third, we observe a stability area, not represented with colours but with hatching in Figure 8b. The BP bifurcations correspond then to limits of global stability like in the two-tube case.

As a summary, our numerical framework provides on one hand the cardinality of three-tube CTR deploying in a FTL manner, which has not been studied until now. On the other hand, it allows to evaluate with minimal implementation efforts a global stability area defined in the actuation input space. Choosing $\Delta L_{2}$ and $\Delta L_{3}$ in this area allows to deploy the robot while maintaining the robot backbone on the reference path. We believe such information is of interest to design stable paths while considering FTL deployment.

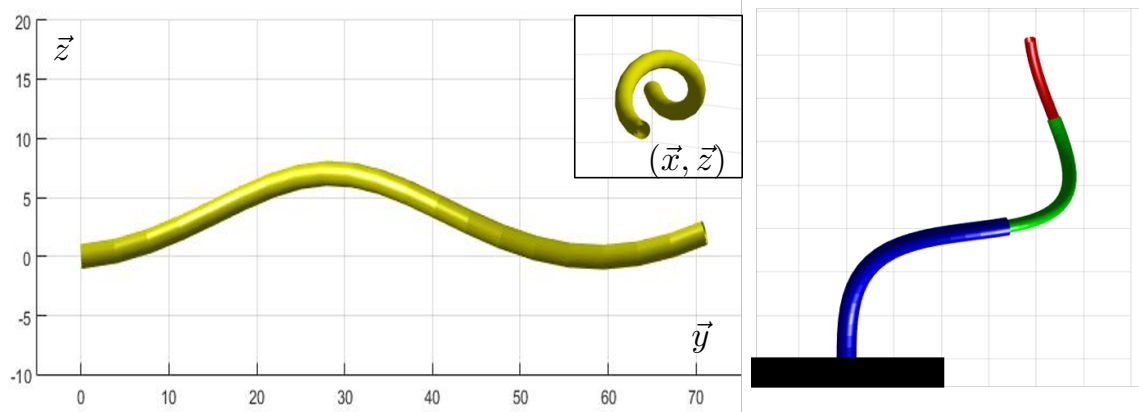

(a) Helical tube.

(b) Telescopic assembly of three helical tubes represented in blue, green and red.

Figure 9: Spatial representation of one helical tube and a CTR composed of tubes with helical shape. 


\subsection{Extension to helical pre-shaped tubes}

Helical tubes are tubes with an initial helical shape as illustrated in Figure $9 \mathrm{a}$. They are defined mathematically by choosing the pre-curvature $\hat{u}_{i z}$ along $\overrightarrow{z_{i}}$ different from zero. This geometrical condition is sufficient to defeat the establishment of current global stability criteria for $n$ tubes CTR [15]. However, this information is useful for the achievement of FTL deployments as explained in the previous sub-section. Our numerical framework is used here to get global stability information on robots composed of helical tubes, such as the one represented in Figure 9b.

We consider the three-tube robot with parameters given in Table 3 for which tubes have the same helical pre-curvature:

$$
\hat{u}_{i z}=\hat{u}_{z} \quad \forall i=1 \ldots n
$$

Three statements are used to ensure the FTL behavior during the deployment. First, the three tubes are mounted in opposition. Second, we consider null transmission lengths, so that the FTL condition concerning the helical tubes actuation given in [8] is respected. It facilitates also the understanding of stability analysis results by reducing the number of parameters involved in the elastic stability. Third, the tubes are deployed following the sequence described in Table 4. We focus here on the first step of the deployment. The corresponding bifurcation diagram is presented on Figure 10a.

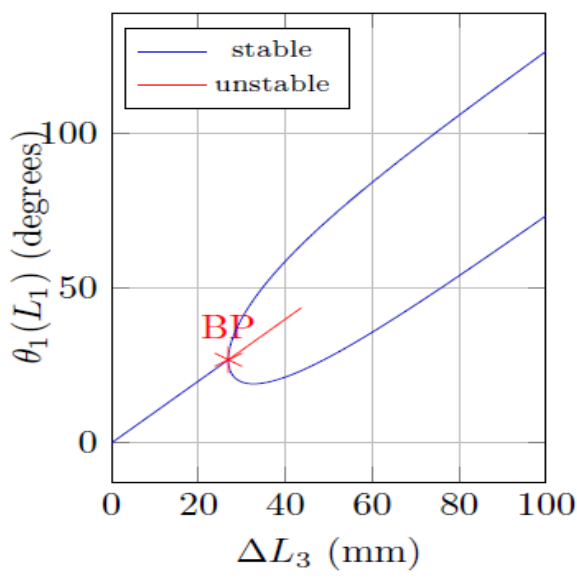

(a) Pitchfork diagram for $\hat{u}_{z}=1 \mathrm{~mm}^{-1}$.

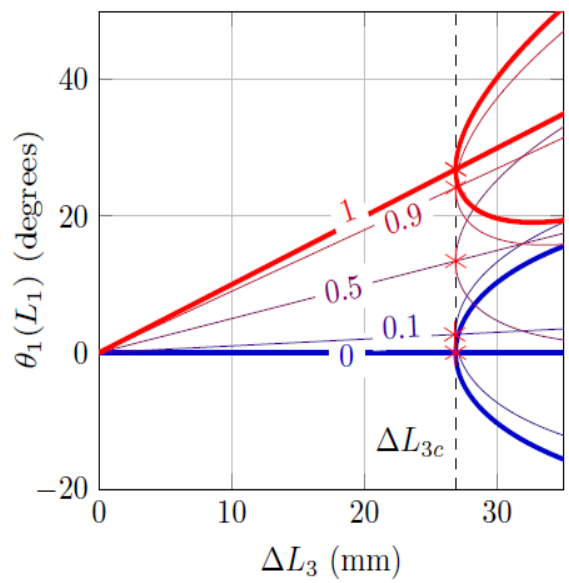

(b) Evolution of the pitchfork diagram with $\hat{u}_{z}$ expressed in $\mathrm{mm}^{-1}$. Values of the precurvature are written in the colour of the corresponding diagram.

Figure 10: Equilibrium branches corresponding to the FTL deployment of CTR composed of three helical tubes. The bifurcations are marked with red stars. 
The length $\Delta L_{3}$ is increased during the branch computation. The tip orientation of tube 1 evolves linearly, with a slope corresponding to the helical pre-curvature, until the equilibrium branch reaches a BP bifurcation. The second resulting branch is then computed in order to obtain the expected pitchfork diagram. The critical length, which represents the global stability limit, corresponds finally to the section length evaluated at the BP. We obtain thus this new result with our generic approach without making additional theoretical or implementation efforts.

In order to go further in the analysis of CTR with helical tubes, we study the impact of helical pre-curvature on the robot cardinality. The critical interaction length is then determined for several values of $\hat{u}_{z}$. This implies to compute several bifurcation diagrams which are superposed on Figure 10b. For the considered case study, the critical interaction length does not seem to depend on the helical pre-curvature, which is a clue for the design of such a CTR.

It is also interesting to observe on Figure 10a that the second branch composing the pitchfork is not symmetric with respect to the first linear branch. This result is somehow difficult to predict considering the behavior of the currently used robot in the literature. The superposed diagrams on Figure 10b show the evolution of the branch asymmetry according to $\hat{u}_{z}$, and the transition between the helical tube and the planar tube cases. As $\hat{u}_{z}$ decreases, the asymmetry disappears progressively until the symmetric pitchfork diagram of CTR with planar tube is reached at $\hat{u}_{z}=0 \mathrm{~mm}^{-1}$.

\section{Conclusion}

We propose in this paper a new numerical framework for the analysis of concentric tube robots stability and cardinality. It is composed of a complete kinematic model of CTR solved with dynamic relaxation and analyzed by continuation and bifurcation methods. The framework is able to simulate CTR deployment, to evaluate local stability and to give information about the number of possible equilibria. It was validated on a reference case study by reproducing bifurcation diagrams given in the literature, with an accuracy depending mainly on the number of points representing the robot. On the opposite of the existing analytical studies, our numerical framework is not limited by kinematic model complexity. Consequently, it has been used to generate new results such as the number of equilibria and the critical length of three-tube CTR deploying in a FTL manner. Moreover, stability analysis of robots composed of helical tubes was performed for the first time.

As perspectives, our numerical framework may be used to study the impact of design parameters on the CTR cardinality and stability instead of actuation inputs. It could specifically provide robot performance sensitivity to these parameters, as well as critical values which ensure elastic stability. This numerical framework may also be used to observe the influence of external forces on the robot behavior, which we think is possible by accounting for these forces in the kinematic model. It may finally be interesting to study with this numerical 
framework other continuum robots, which are subject to elastic instabilities and which behavior is also described by a BVP.

\section{Acknowledgement}

This work was supported by the French National Agency for Research within the Biomedical Innovation program (NEMRO ANR-14-CE17-0013), the Investissements d'Avenir (Robotex ANR-10-EQPX-44, Labex CAMI ANR-11LABX-0004 and EUR EIPHI (Contract No. ANR-17-EURE-0002)) and Aviesan France Life Imaging infrastructure. 


\section{References}

[1] J. Burgner-Kahrs, D. C. Rucker, and H. Choset. Continuum Robots for Medical Applications: A Survey. IEEE Transactions on Robotics, 31(6):1261-1280, December 2015.

[2] X. Chen, C. Zheng, W. Xu, and K. Zhou. An Asymptotic Numerical Method for Inverse Elastic Shape Design. ACM Trans. Graph., 33(4):95:195:11, July 2014.

[3] Howie Choset and Wade Henning. A Follow-the-Leader Approach to Serpentine Robot Motion Planning. Journal of Aerospace Engineering, 12(2):65-73, April 1999.

[4] A. Dhooge, W. Govaerts, Yu.A. Kuznetsov, W. Mestrom, A.M. Riet, and B. Sautois. MATCONT and CL_matcont: Continuation toolboxes in MATLAB. Manual, Universiteit Gent, Utrecht University, Belgium, The Netherlands, December 2006.

[5] E.J. Doedel. AUTO, a program for the automatic bifurcation analysis of autonomous systems. Cong. Numer., (30):265-384, 1981.

[6] P. E. Dupont, J. Lock, B. Itkowitz, and E. Butler. Design and Control of Concentric-Tube Robots. IEEE Transactions on Robotics, 26(2):209-225, April 2010.

[7] H. B. Gilbert, R. J. Hendrick, and R. J. Webster III. Elastic Stability of Concentric Tube Robots: A Stability Measure and Design Test. IEEE Transactions on Robotics, 32(1):20-35, February 2016.

[8] H. B. Gilbert, J. Neimat, and R. J. Webster. Concentric Tube Robots as Steerable Needles: Achieving Follow-the-Leader Deployment. IEEE Transactions on Robotics, 31(2):246-258, April 2015.

[9] C. Girerd, K. Rabenorosoa, and P. Renaud. Synthesis of a New Concentric Tube Robot for Olfactory Cells Exploration. In Computer/Robot Assisted Surgery (CRAS), September 2016.

[10] C. Girerd, K. Rabenorosoa, and P. Renaud. Combining Tube Design and Simple Kinematic Strategy for Follow-the-Leader Deployment of Concentric Tube Robots. In Advances in Robot Kinematics 2016, volume 4, pages 23-31. Springer, Cham, 2018.

[11] A. H. Gosline, N. V. Vasilyev, E.J. Butler, C. Folk, A. Cohen, R. Chen, N. Lang, P. J. del Nido, and P. E. Dupont. Percutaneous intracardiac beating-heart surgery using metal MEMS tissue approximation tools. The International journal of robotics research, 31(9):1081-1093, August 2012. 
[12] A. V. P. Gurumoorthy and J. R. Picardo. Evaluation of MATCONT of MATLAB for Constructing Bifurcation Diagrams of Chemical Process Systems. SSRN Scholarly Paper ID 2100637, Social Science Research Network, Rochester, NY, September 2011.

[13] J. Ha, F. C. Park, and P. E. Dupont. Achieving elastic stability of concentric tube robots through optimization of tube precurvature. In 2014 IEEE/RSJ International Conference on Intelligent Robots and Systems, pages 864-870, Chicago, September 2014.

[14] J. Ha, F. C. Park, and P. E. Dupont. Elastic Stability of Concentric Tube Robots Subject to External Loads. IEEE Transactions on Biomedical Engineering, 63(6):1116-1128, June 2016.

[15] R. J. Hendrick, H. B. Gilbert, and R. J. Webster. Designing snap-free concentric tube robots: A local bifurcation approach. In 2015 IEEE International Conference on Robotics and Automation (ICRA), pages 2256-2263, Seattle, May 2015.

[16] R. J. Webster III, J. M. Romano, and N. J. Cowan. Mechanics of PrecurvedTube Continuum Robots. IEEE Transactions on Robotics, 25(1):67-78, February 2009.

[17] D. Jo and E. J. Haug. Workspace Analysis of Multibody Mechanical Systems Using Continuation Methods. Journal of Mechanisms, Transmissions, and Automation in Design, 111(4):581-589, December 1989.

[18] Y. A. Kuznetsov. Numerical Analysis of Bifurcations. In Elements of Applied Bifurcation Theory, volume 112 of Applied Mathematical Sciences, pages 505-585. Springer, New York, NY, 2004.

[19] Y. A. Kuznetsov. One-Parameter Bifurcations of Equilibria in ContinuousTime Dynamical Systems. In Elements of Applied Bifurcation Theory, volume 112 of Applied Mathematical Sciences, pages 77-115. Springer, New York, NY, 2004.

[20] A. Lazarus, J. T. Miller, and P. M. Reis. Continuation of equilibria and stability of slender elastic rods using an asymptotic numerical method. Journal of the Mechanics and Physics of Solids, 61(8):1712-1736, August 2013.

[21] D. Y. Lee, J. Kim, J. S. Kim, C. Baek, G. Noh, D. N. Kim, K. Kim, S. Kang, and K. J. Cho. Anisotropic Patterning to Reduce Instability of ConcentricTube Robots. IEEE Transactions on Robotics, 31(6):1311-1323, December 2015 .

[22] K. Leibrandt, C. Bergeles, and G. Z. Yang. Implicit Active Constraints for Safe and Effective Guidance of Unstable Concentric Tube Robots. In IEEE/RSJ International Conference on Intelligent Robots and Systems (IROS), Deajon, October 2016. 
[23] F. Y. Lin, C. Bergeles, and G. Z. Yang. Biometry-based concentric tubes robot for vitreoretinal surgery. In 2015 37th Annual International Conference of the IEEE Engineering in Medicine and Biology Society (EMBC), pages 5280-5284, August 2015.

[24] A. Najah, B. Cochelin, N. Damil, and M. Potier-Ferry. A critical review of asymptotic numerical methods. Archives of Computational Methods in Engineering, 5(1):31-50, March 1998.

[25] J. Olsson. Form finding and size optimization - Implementation of beam elements and size optimization in real time form finding using dynamic relaxation. Master thesis, Chalmers University of Technology, Gothengurg, Sweden, 2012.

[26] Q. Peyron, I. Charpentier, and E. Laroche. Continuation for Stability Domain Determination with Application to a Cable-Driven Parallel Robot. In World Congress of the International Federation of Automatic Control, Toulouse, July 2017.

[27] W. H. Press, S. A. Teukolsky, W. T. Vetterling, and B. P. Flannery. Numerical Recipes in $C$. The Art of Scientific Computing. Cambridge University Press, second edition edition, 2002.

[28] J. Rodriguez, G. Rio, J. M. Cadou, and J. Troufflard. Numerical study of dynamic relaxation with kinetic damping applied to inflatable fabric structures with extensions for 3d solid element and non-linear behavior. Thin-Walled Structures, 49(11):1468-1474, November 2011.

[29] D. C. Rucker, R. J. Webster, G. S. Chirikjian, and N. J. Cowan. Equilibrium Conformations of Concentric-tube Continuum Robots. The International Journal of Robotics Research, 29(10):1263-1280, September 2010. 


\section{A. Example of simulation code for CL-MatCont}

1\% Deployment simulation of section 3 for a three-tube CTR. The kinematic model is written in the System file called "discrete_CTR_system.m".

$\%$ Initialization of CL-MatCont toolbox

init;

\% Loading of CTR parameters, comprinsing the number of tube, the section length, their geometry and their mechanical properties. In this case

${ }_{6} \% \mathrm{p}=[\mathrm{Ni}$, Delta Li, kit, kib, uix, uiy, uiz $], \mathrm{i}=1,2,3$

$7 \mathrm{p}=\mathrm{CTR} \_$parameters;

\% Computation of the initial configuration of the robot with dynamic relaxation. X0 contain the twist angles of each tube evaluated at each point on the robot backbone.

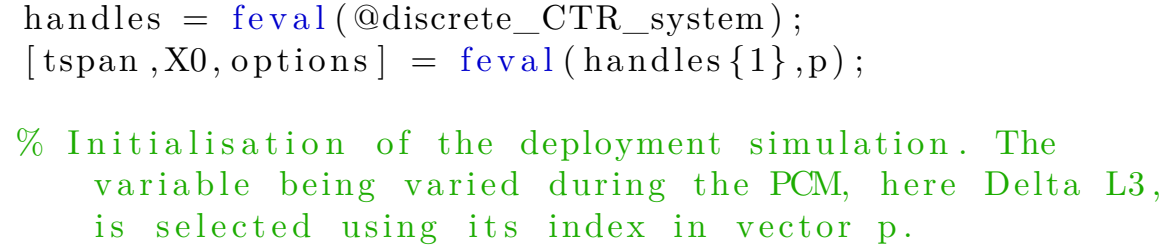


the steps contained in the CL-MatCont block of the flow chart diagram presented in Figure 4.

$31[\mathrm{x}, \mathrm{v}, \mathrm{s}, \mathrm{h}, \mathrm{f}]=\operatorname{cont}(@$ equilibrium $, \mathrm{x} 1, \mathrm{v} 1, \mathrm{opt}) ;$ 